\title{
The geometry of the Barbour-Bertotti theories I. The reduction process
}

\author{
László Á Gergely †‡ \\ $\dagger$ Laboratoire de Physique Théorique, Université Louis Pasteur, 3-5 rue de l'Université \\ 67084 Strasbourg, France \\ $\ddagger$ KFKI Research Institute for Particle and Nuclear Physics, Budapest 114, P.O.Box \\ 49, H-1525 Hungary
}

\begin{abstract}
The dynamics of $N \geq 3$ interacting particles is investigated in the non-relativistic context of the Barbour-Bertotti theories. The reduction process on this constrained system yields a Lagrangian in the form of a Riemannian line element. The involved metric, degenerate in the flat configuration space, is the first fundamental form of the space of orbits of translations and rotations (the Leibniz group). The Riemann tensor and the scalar curvature are computed by a generalized Gauss formula in terms of the vorticity tensors of generators of the rotations. The curvature scalar is further given in terms of the principal moments of inertia of the system. Line configurations are singular for $N \neq 3$. A comparison with similar methods in molecular dynamics is traced.
\end{abstract}

\section{Introduction}

Since the very gestation of the Newtonian mechanics [1], severe criticism of its concepts of absolute space and time has proliferated. The criticism concerning absolute space, as the tool determining the privileged inertial frames is best expressed by Mach's principle [2]: inertial frames are an imprint of the matter content of the universe. According to Leibniz [3], time is nothing more than the sequence of events. Some of these ideas were incorporated in the general theory of relativity. There, time is not absolute and Mach's principle is satisfied at least for closed universes 田, 5. The generic idea of timelessness of both a non-relativistic and a general relativistic complete universe was analyzed recently by Barbour [6].

In this generic framework there were also several attempts to incorporate Mach's principle and Leibniz's concept of time in dynamical theories at the non-relativistic level. Newtonian absolute space is eliminated from such models and in some of them absolute time too, by implementing time reparametrization invariance. However, in contrast with general relativity, these models retain absulute simultaneity. The study of such models dates back to the early attempts of Reißner [7, 8], who has considered the dynamics of the system of $N$ interacting particles which depend on $N(N-1) / 2$ mutual distances.

In the model developed by Lynden-Bell [9] both the kinetic and the potential term in the Lagrangian are invariant under time-dependent translations and rotations. This theory, as described by Lynden-Bell and Katz 4 is equivalent with Newtonian mechanics in a universe of zero net angular momentum about its barycenter, provided the latter is in uniform motion. There exists a strong evidence for the assumption 
of vanishing angular momentum of the universe, given by the analysis of the cosmic microwave background radiation [10, 11, 12], in support to the validity of the theory. Lynden-Bell's model describes an $N$-body system of interacting particles in terms of purely relative quantities. No overall (time-dependent) rotation of the frame about the barycenter or translational motion of the barycenter has any effect on the action functional or the equations of motion derived from it. This happens because no other distances and velocities appear in the action than the distances between pairs of particles and the differences of angular velocities of the lines joining these pairs of particles, respectively. General relativistic extensions of these ideas were discussed by Lynden-Bell, Katz and Bičak [5]. In Lynden-Bell's model, however, time is absolute.

The fully relational dynamical models introduced by Barbour and Bertotti 13, 14] are more general than Lynden-Bell's theory in bearing invariance with respect to the whole Leibniz group 13] (including a monotonic redefinition of the time):

$$
\begin{aligned}
& x^{a^{\prime}}=\mathcal{R}_{b}^{a^{\prime}}(t) x^{b}+\Lambda^{a^{\prime}}(t) \\
& t^{\prime}=t^{\prime}(t) .
\end{aligned}
$$

Here $\mathcal{R}$ is an orthogonal matrix and $\Lambda^{a^{\prime}}$ are arbitrary translation parameters. As a consequence of employing an arbitrary time parameter, the Lagrangian for a system of $N$ particles becomes a homogeneous function of degree one in the "velocities" $\left(d q_{i} / d t^{\prime}, d t / d t^{\prime}\right)$, implying via Euler's theorem a vanishing Hamiltonian for the system [15. As an immediate consequence of this and the invariance (1) seven constants of the motion identically vanish. These are the energy, the linear and angular momenta. Barbour and Bertotti start from "the relative motion of the universe treated as a single entity and then recover the motion of subsystems within the background provided by the universe at large". The particular product Lagrangian proposed in [13] although being able to (qualitatively) reproduce some general relativistic effects, like the perihelion shift and the Lense-Thirring effect, leads also to unpleasant consequences: anisotropic masses (in contradiction with experiment), time-dependent gravitational constant and a violation of the Birkhoff theorem. In their second paper, Barbour and Bertotti 14 have proposed a more generic framework for deriving intrinsic dynamics, relying on the concept of intrinsic differential. Both Galilei- and Lorentz-invariance could be derived for particular subsystems of the whole Leibniz-invariant universe.

As the theories of Barbour and Bertotti share with the theory of general relativity the property of possessing both the Hamiltonian and momenta constraints, the study of these models, particularly the issues concerning quantization, can give an insight of how such theories should be handled in a quantization process. Particularly interesting are the solutions given to conceptual and interpretational difficulties by Barbour and Smolin [16, where an explicit solution is presented for the case of three particles in one dimension. In a second paper [17], Barbour and Smolin introduce the concept of variety in order to provide a (non-local) description of dynamical systems without any reference to elements lying outside the domain of valability of these theories. The Barbour-Bertotti theories are among the toy-models on which the applicability of their new concept and principle are checked. A group-theoretical quantization of the Barbour-Bertotti theories was done by Rovelli [18], eliminating any concept of time. In contrast, an approach relying on a quantization in terms of an intrinsic time was performed by Gambini and Mora [19. Arguing that time is a manifestation of dynamics, Barbour arrives to the notion of timelessness of quantum gravity [6] and presents an approach to recover time from a static wavefunction of the universe [20]. We will not enter in the details of the quantization process here. Our aim in this 
and the subsequent companion paper [21] is to analyze several aspects related to the geometric structure of these theories.

In what follows, by a Barbour-Bertotti type theory we mean a nonrelativistic dynamical theory obeying the Hamiltonian, momenta and angular momenta constraints. In Sec. 2 we start from the Newtonian description of the interacting $N$-particle system, imposing the above constraints. Then, by solving the constraints and eliminating the Lagrange-multipliers we obtain the reduced Lagrangian, homogeneous in the velocities.

This action determines a Riemannian metric, in a form of a line element of some Riemannian space. Extremizing the action is equivalent with finding the geodesic motions. These issues are discussed in Sec. 3. Although the particular form of the Lagrangian differs from that given in $\sqrt{13}$, it has a product structure, in accordance with the general framework settled in $[13,14]$. What we recover is the Jacobi principle for the constrained $N$-particle system, as expected from the argumentation in [6]. General relativity, in the form given by Baierlein, Sharp and Wheeler 22] resembles also a Jacobi principle rather than a parametrized particle dynamics [6].

In Sec. 4. we study the geometry of the reduced space for the generic case of $N \geq 3$ particles. (For $N=1$ there is no relative distance at all. For $N=2$, as the Lagrangian is homogeneous of degree one in the single relative velocity, the EulerLagrange equation is trivially satisfied and there is again no relative dynamics [13].) For $N \geq 3$ we find that the metric is degenerate and the degeneracy directions are related to the generators of translations and of rotations in the physical space. This is again in accordance with [13, 14, where dynamics is defined on the space of orbits. We compute the curvature scalar of the $3 N-6$ dimensional reduced space (the space of orbits) by means of a generalized Gauss equation, deduced here. The curvature scalar is expressed in terms of the principal moments of inertia of the system. This allows us to conclude that the line configurations are curvature singularities for $N \neq 3$. We will investigate the $N=3$ case in detail in the companion paper [21].

There are certain connections of our work with the analysis of the $N$-body problem by geometric methods in molecular dynamics. A recent summary of this topic was given by Littlejohn and Reinsch [25]. We trace a comparison with their results in Sec. 5 .

There are several type of indices appearing in our formulae. The coordinates of the configuration space are labeled by a particle index, denoted by a capital letter running from 1 to $N$, and a Cartesian coordinate index of the physical space (abstract index), denoted by a Latin letter, which takes the values $1,2,3$. We adopt the convention to write the summation over particles explicitly and apply the summation convention for generic coordinate indices. The (Latin) indices of the Kronecker delta $\delta_{a b}$ and of the totally antisymmetric Levi-Civita symbol $\epsilon_{a b c}$ are raised when necessary in order the summation convention to apply. Kinematical and dynamical quantities written in a system of coordinates originating in the center of mass carry a distinguishing zero subscript, like ${ }_{0}{ }^{A c}$. There is one exception over this rule: the principal moments of inertia $I_{\mu}$ are written without the zero subscript. Greek letters label coordinates in the system of principal axes of inertia, with the origin in the center of mass. The summation over these indices will be also explicitly indicated. 


\section{The reduced Lagrangian of the Barbour-Bertotti model}

We start from the canonical form of the action characterizing a system of $N$ interacting particles:

$$
S[x, p]=\int d t^{\prime}\left(\sum_{A=1}^{N} p_{A a} \frac{d x^{A a}}{d t^{\prime}}-H\right) .
$$

where $x^{A a}, d x^{A a} / d t^{\prime}$ and $p_{A a}$ are the coordinates, velocity and momenta components, respectively of the particle with mass $m_{A}$, and the Hamiltonian is given by:

$$
H=\sum_{A=1}^{N} \frac{1}{2 m_{A}} p_{A a} p_{A b} \delta^{a b}+V
$$

The potential term $V$ is a superposition of the two-particle potentials $V_{A B}$, which depend only on the relative separation between particles $A$ and $B$ :

$$
V=\sum_{A<B} V_{A B}\left(\left|\mathbf{r}^{A}-\mathbf{r}^{B}\right|\right)
$$

$\mathbf{r}^{A}=\left\{x^{A a}\right\}$ being the position vector of particle $A$.

The Hamiltonian (3) is time-independent, implying a conserved energy. The total linear and angular momenta

$$
P_{a}=\sum_{A=1}^{N} p_{A a}, \quad L_{a}=\sum_{A=1}^{N} \epsilon_{a b}^{c} x^{A b} p_{A c}
$$

are also conserved:

$$
\left\{P_{a}, H\right\}=0, \quad\left\{L_{a}, H\right\}=0 .
$$

The above constants of the motion form a closed algebra:

$$
\left\{P_{a}, P_{b}\right\}=0, \quad\left\{L_{a}, L_{b}\right\}=\epsilon_{a b}{ }^{c} L_{c}, \quad\left\{P_{a}, L_{b}\right\}=\epsilon_{a b}{ }^{c} P_{c}
$$

The last group of the above Poisson brackets bears the message that the total momenta transform as vectors under rotations.

The Barbour-Bertotti theory is obtained from the above Newtonian theory by imposing the constraintst:

$$
P_{a}=0, \quad L_{a}=0, \quad H=0 .
$$

Among them, the condition $P_{a}=0$ is fulfilled by any system if the origin of the frame is chosen in the center of mass. The other two constraints do not hold in generic situations, for example in the case of an interacting system of harmonic oscillators, where the energy is positive definite. For attractive forces $H=0$ is the marginally bounded situation.

We add to the canonical action (2) the linear and angular momenta constraints (8) with Lagrange multipliers $\mathcal{N}^{a}$ and $\mathcal{M}^{a}$. By replacing the time $t^{\prime}$ with a labeling parameter $t$ [15], the Hamiltonian also gets a multiplier $\mathcal{N}$ :

$$
S\left[x, p ; \mathcal{N}, \mathcal{N}^{a}, \mathcal{M}^{a}\right]=\int d t\left(\sum_{A=1}^{N} p_{A a} \dot{x}^{A a}-\mathcal{N} H-\mathcal{N}^{a} P_{a}-\mathcal{M}^{a} L_{a}\right) .
$$

$\dagger$ Without imposing $H=0$ and when $V_{A B}$ are the Newtonian gravitational potentials, the LyndenBell theory 99 is recovered. 
(The notation $\dot{x}^{A a}=d x^{A a} / d t$ was introduced.) Variations of this action as a functional of the multipliers generate the constraints. Variations of (9) regarded as functional of the coordinates and momenta give the equations of motion, equivalent with the Newtonian equations for a system satisfying the constraints.

Next we proceed with the reduction of the action by solving the constraints and expressing the Lagrange multipliers as functions of configuration data. First by varying $p_{A a}$ in (9) we find the relation between the velocities and linear momenta:

$$
\dot{x}^{A a}=\mathcal{N} \frac{p_{A b}}{m_{A}} \delta^{a b}+\mathcal{N}^{a}-\mathcal{M}_{b}^{a} x^{A b} .
$$

Here we have introduced the dualized form of $\mathcal{M}^{a}$, defined by the relations:

$$
\mathcal{M}_{a b}=\epsilon_{a b c} \mathcal{M}^{c}, \quad \mathcal{M}^{a}=\frac{1}{2} \epsilon^{a b c} \mathcal{M}_{a b} .
$$

The unusual relation (10) between the velocities and momenta is consequence of the constrained nature of the system (9). From Eq. (10) we express the momenta as:

$$
p_{A a}=\mathcal{N}^{-1} m_{A}\left(\dot{x}^{A b} \delta_{a b}-\mathcal{N}_{a}+\mathcal{M}_{a b} x^{A b}\right) .
$$

The total linear momentum of the system is found by summing up the above momenta over all particles:

$$
P_{a}=\mathcal{N}^{-1} M\left(\dot{x}^{b} \delta_{a b}-\mathcal{N}_{a}+\mathcal{M}_{a b} x^{b}\right) .
$$

Here $M=\sum_{A=1}^{N} m_{A}$ is the total mass of the system, $x^{a}=\sum_{A=1}^{N} m_{A} x^{A a} / M$ and $\dot{x}_{a}$ are the coordinates and the velocities of the center of mass, respectively.

In a similar manner by summing up over all particles the angular momenta $l_{A a}=\epsilon_{a b}{ }^{c} x^{A b} p_{A c}$, the total angular momentum emerges:

$$
L_{a}=\mathcal{N}^{-1}\left(l_{a}-I_{a b} \mathcal{M}^{b}-M \mathcal{N}_{a b} x^{b}\right) .
$$

In the above expression we have introduced the velocity-based total angular momentum:

$$
l_{a}=\epsilon_{a b c} \sum_{A=1}^{N} m_{A} x^{A b} \dot{x}^{A c}
$$

the tensor of inertia:

$$
I_{g h}=\epsilon_{c g}^{l} \epsilon_{l d h} \sum_{A=1}^{N} m_{A} x^{A c} x^{A d}
$$

and the dualized Lagrange-multiplier $\mathcal{N}_{a b}$ related to $\mathcal{N}^{a}$ as:

$$
\mathcal{N}_{a b}=\epsilon_{a b c} \mathcal{N}^{c}, \quad \mathcal{N}^{a}=\frac{1}{2} \epsilon^{a b c} \mathcal{N}_{a b}
$$

By virtue of the constraints (8) the equations (13) and (14) form an inhomogeneous linear algebraic system in the multipliers $\mathcal{N}^{a}$ and $\mathcal{M}^{a}$. We transform this system to an equivalent form by expressing the multipliers $\mathcal{N}_{a}$ from (13) then inserting them into (14) obtaining:

$$
\mathcal{N}_{a}=\mathcal{M}_{a b} x^{b}+\dot{x}^{b} \delta_{a b}, \quad I_{\circ} a b \mathcal{M}^{b}=l_{\circ} a
$$


Here

$$
\begin{aligned}
& \underbrace{x^{A a}}_{\circ}=x^{A a}-x^{a}=x^{A a}-\frac{1}{M} \sum_{A=1}^{N} m_{A} x^{A a} \\
& {\underset{\circ}{\circ} a}_{a}=\epsilon_{a b c} \sum_{A=1}^{N} m_{A} x_{\circ}^{A b} \dot{x}_{\circ}^{A c} \\
& \underset{\circ}{I} g h=\epsilon_{c g}^{l} \epsilon_{l d h} \sum_{A=1}^{N} m_{A} \underset{\circ}{x_{\circ}^{A c} x_{\circ}^{A d}}
\end{aligned}
$$

are the coordinates, the velocity-based angular momentum and the inertia tensor in the center of mass frame, respectively.

There is a solution of the second equation (18) only when the tensor of inertia in the center of mass frame is nondegenerate $\dagger$. A careful study shows that a degenerate inertia tensor would only occur in the case of all $N$ particles being along a line (giving a rank 2 inertia tensor) or in the unphysical situation of all particles at the same point (rank 0 inertia tensor). The inverse inertia tensor $\left(I^{-1}\right)^{a b}$ is well defined for all other configurations of the particles. Thus, modulo the above exceptional cases, the multipliers $\mathcal{M}^{a}$ are:

$$
\mathcal{M}^{a}=\left(I^{-1}\right)^{a b} l_{\circ} b
$$

If we insert the expressions of the multipliers $\mathcal{N}^{a}$ and $\mathcal{M}^{a}$ from (18) and (22) in the expression of the momenta $(12)$ we obtain:

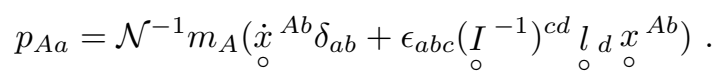

We have solved the linear and angular momenta constraints, thus the terms containing the multipliers $\mathcal{N}^{a}$ and $\mathcal{M}^{a}$ can be dropped from the action (9). By inserting the expression of the momenta (23), the remaining Liouville form and lapseHamiltonian term become:

$$
\begin{aligned}
& \sum_{A=1}^{N} p_{A a} \dot{x}^{A a}=2 \mathcal{N}^{-1}\left(\underset{\circ}{T}-\frac{1}{2} I_{\circ}{ }_{a b} \mathcal{M}^{a} \mathcal{M}^{b}\right) \\
& \mathcal{N} H=\mathcal{N}^{-1}\left(\underset{\circ}{T}-\frac{1}{2} \underset{\circ}{I_{a b}} \mathcal{M}^{a} \mathcal{M}^{b}\right)+\mathcal{N} V .
\end{aligned}
$$

Here $\underset{\circ}{T}$ is the kinetic energy in the center of mass frame:

$$
\underset{\circ}{T}=\frac{1}{2} \sum_{A=1}^{N} m_{A} \underset{\circ}{\dot{x}_{\circ}^{A a}} \underset{\circ}{\dot{x}^{A b}} \delta_{a b}
$$

The action is now a functional on the configuration space and function of the lapse alone:

$$
S[x ; \mathcal{N}]=\int d t L, \quad L(x, \dot{x} ; \mathcal{N})=\mathcal{N}^{-1}\left(\underset{\circ}{T}-\frac{1}{2} \underset{\circ}{I_{a b}} \mathcal{M}^{a} \mathcal{M}^{b}\right)-\mathcal{N} V
$$

$\dagger$ One's restricted ability in expressing the Lagrange multipliers in terms of the configuration data arises also in the thin sandwich conjecture 22 of general_relativity. The local solvability of that problem for a large class of initial data was proved in 23. Recently results on the existence of solutions of the generalized thin-sandwich problem were also obtained 24 . 
The variation with respect to $\mathcal{N}$ gives an equation which we use to express the lapse:

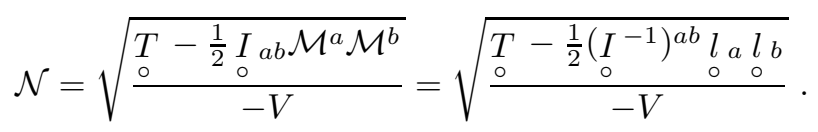

By eliminating all multipliers, the task of reducing the action is completed:

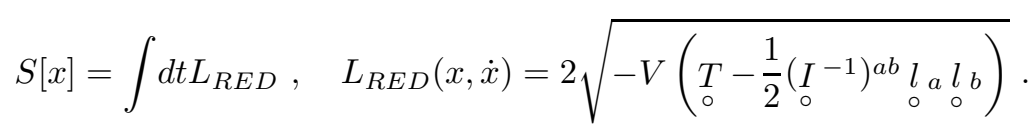

We have obtained the Jacobi Principle version of Lynden-Bell's purely relative Lagrangian with the energy set to zero.

\section{The induced geometry}

The action (29) obtained by completing the reduction process (for all noncollinear configurations) is homogeneous of degree one in the velocities. Therefore the integrand can be regarded as a line element in some Riemannian space. In order to make this manifest, we write the reduced Lagrangian 29 in two alternative forms:

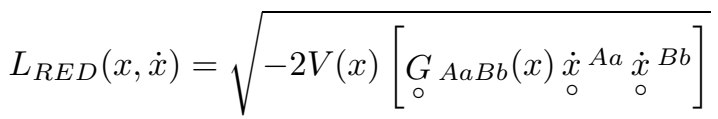

$$
\begin{aligned}
& =\sqrt{-2 V(x)\left[G_{A a B b}(x) \dot{x}^{A a} \dot{x}^{B b}\right]},
\end{aligned}
$$

where $\underset{\circ}{G A a B b}$ and $G_{A a B b}$ are Riemannian metrics defined as:

$$
\begin{aligned}
& \underset{\circ}{G A a B b}=m_{A} \delta_{A B} \delta_{a b}-m_{A} m_{B}\left(\stackrel{\circ}{I}^{-1}\right)^{e f} \epsilon_{e c a} \epsilon_{f d b} \underset{\circ}{{ }_{\circ}}{ }_{\circ}^{A c} \underset{\circ}{x}{ }^{B d} \\
& G_{A a B b}=G_{\circ} A a B b-\frac{m_{A} m_{B}}{M} \delta_{a b} .
\end{aligned}
$$

Without changing the notations, from now on an index pair $A a$ is viewed as a single index running from 1 to $3 N$. Though the reduction process at the Lagrangian level was accomplished, the configuration space is still $3 N$-dimensional. It will be one of the tasks of the next section to show that the $3 N$-dimensional configuration space is superfluous and the $(3 N-6)$-dimensional space of orbits of the Leibniz group is better suited as a configuration space.

The metric has the simpler form (31) in the center of mass frame, however this expression is covariant only under rotations in the physical space. In contrast, the expression (32), containing an additional term, is covariant under both translations and rotations in the physical space. It can be brought into a completely generic form by inserting the expressions (19) of the coordinates $\underset{\circ}{x}{ }^{A c}$ in $(32)$ and in $\left(I_{0}^{-1}\right)^{d f}$ appearing there.

The geometry underlying the physical motions is characterized by the conformally scaled (Jacobi) metric $-2 V(x) G_{A a B b}$. Extremizing $L_{R E D}$ is equivalent with looking for the geodesic motions associated with this metric. Dynamics arise from geometry. To our knowledge it was Synge [26] who first applied this viewpoint in a discussion of conservative systems in the framework of a "geometro-dynamical theory of the manifold of configurations". A recent discussion of the Jacobi metric for simple 
dynamical systems [27] concentrates on the singular regions at $E=V$ (at $V=0$ when specialized to our case).

As $V(x)$ varies from case to case, in the remaining part of the paper we investigate the Riemannian metric (32). Formally, the metric $G_{A a B b}$ is characterizing the free motions pertinent to the $V=$ const case.

At the end of this section we stress that the elimination of the multiplier $\mathcal{N}$ from the action (27) implies a choice of time. This is because the elimination was carried out by employing $\delta S[x ; \mathcal{N}] / \delta \mathcal{N}$ which is the Hamiltonian constraint $H=T+V=E=0$. However in a relational theory no time exists a priori, in consequence the energy conservation equation is not giving the speed of the system on its trajectory in the configuration space, as usually. Rather it defines a unic time $t$ for all subsystems [6].

\section{The reduction to the space of orbits}

For infinitesimal translations and rotations $d x^{A a}=d \xi^{a}+\epsilon_{b c}^{a} x^{A b} d \eta^{c}$ we have $G_{A a B b} d x^{A a}=0$. Therefore the metric is degenerated, the directions of degeneracies being the vector flows in the configuration space induced by translations and rotations in the physical space.

With respect to the flat metric

$$
g_{A a B b}=m_{A} \delta_{A B} \delta_{a b}, \quad g^{A a B b}=\frac{1}{m_{A}} \delta^{A B} \delta^{a b},
$$

the generators of translations $\xi_{(i)}^{B b}=\delta_{i}^{b}$ and of rotations $\eta_{(j)}^{B b}=\epsilon_{c j}^{b} x^{B c}$ do not form an orthogonal set:

$$
\begin{aligned}
& \sum_{A, B=1}^{N} g_{A a B b} \xi_{(i)}^{A a} \xi_{(j)}^{B b}=M \delta_{i j} \\
& \sum_{A, B=1}^{N} g_{A a B b} \xi_{(i)}^{A a} \eta_{(j)}^{B b}=\epsilon_{i c j} x^{c} \\
& \sum_{A, B=1}^{N} g_{A a B b} \eta_{(i)}^{A a} \eta_{(j)}^{B b}=I_{i j}
\end{aligned}
$$

excepting the case when the coordinate axes originate in the center of mass $\left(x^{c}=0\right.$ and $I_{i j}=I_{0} j$ ) and they are chosen along the principal axes of the inertia tensor (where $I_{\circ}=I_{\mu} \delta_{\mu \nu}, I_{\mu}$ denoting the principal moments of inertia). In such a system an orthonormal set of six generators with respect to the flat metric (33) is given by:

$$
z_{(\mu)}^{B \beta}=\frac{1}{\sqrt{M}} \delta_{\mu}^{\beta}, \quad w_{(\nu)}^{B \beta}=\frac{1}{\sqrt{I_{\nu}}} \epsilon_{\gamma \nu}^{\beta}{\underset{\circ}{0}}^{B \gamma} .
$$

The generators (35) correspond to translations along, and rotations about the principal axes of inertia, originating in the center of mass. The dual set of these generators is:

$$
u_{A \alpha}^{(\mu)}=\frac{m_{A}}{\sqrt{M}} \delta_{\alpha}^{\mu}, \quad v_{A \alpha}^{(\nu)}=\frac{m_{A}}{\sqrt{I_{\nu}}} \epsilon_{\alpha \gamma}^{\nu} x_{\circ}^{A \gamma} .
$$

The expressions of the generators in generic frames are obtained by the tensor 
transformation law:

$$
\begin{aligned}
& z_{(\mu)}^{B b}=\sum_{C \gamma} z_{(\mu)}^{C \gamma} \frac{\partial x^{B b}}{\partial x_{\circ}^{C \gamma}}, \quad w_{(\nu)}^{B b}=\sum_{C \gamma} w_{(\nu)}^{C \gamma} \frac{\partial x^{B b}}{\partial x_{\circ}^{C \gamma}}
\end{aligned}
$$

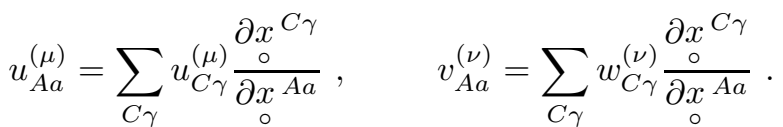

We conclude that $G_{A a B b}$ is the metric in the $(3 N-6)$ dimensional space of orbits of the group of translations and rotations, rigged by the generators $(37) \dagger$ :

$$
G_{A a B b}=g_{A a B b}-\sum_{\mu} u_{A a}^{(\mu)} u_{B b}^{(\mu)}-\sum_{\nu} v_{A a}^{(\nu)} v_{B b}^{(\nu)} .
$$

We define

$$
P_{B b}^{A a}=\delta_{B}^{A} \delta_{b}^{a}-\sum_{\mu} z_{(\mu)}^{A a} u_{B b}^{(\mu)}-\sum_{\nu} w_{(\nu)}^{A a} v_{B b}^{(\nu)} .
$$

It is an easy exercise to verify that $P_{B b}^{A a}$ is a projection operator

$$
P_{C c}^{A a} P_{B b}^{C c}=P_{B b}^{A a}
$$

to the subspace rigged by the generators:

$$
P_{B b}^{A a} z_{(\mu)}^{B b}=P_{B b}^{A a} u_{A a}^{(\mu)}=0, \quad P_{B b}^{A a} w_{(\nu)}^{B b}=P_{B b}^{A a} v_{A a}^{(\nu)}=0 .
$$

The construction we have found is quite generic. Whenever one has a Riemannnian manifold and a gauge group of isometries acting on it, the metric on the quotient space is naturally given by the restriction of the initial metric to the orthogonal complement to the gauge group. We stress that none of $w_{(\nu)}^{B b}$ and $v_{A a}^{(\nu)}$ and neither $G_{A a B b}$ are welldefined when any of the principal moments of inertia vanish. Thus we have to limit the validity of the above construction to the noncollinear configurations. The reduced space is the quotient space $R^{3 N} / E(3)$ where $E(3)=R^{3} \rtimes S O(3)$. By extending this manifold to include the collinear configurations either, we find a manifold with dimensionality $3 N-6$ with boundary for $N=3$ and without boundary for $N \geq 4$ [25]. The case $N=3$ will be discussed in detail in the companion paper [21]. For $N=4$ the manifold in discussion is homeomorphic with $R^{6}$ [29].

We denote by $\nabla$ and $\tilde{\nabla}$ the connections compatible with the metric $G$ and the flat metric $g$. The respective covariant derivatives of an arbitrary vector field $q^{A a}$ in the coordinate basis $\left\{\frac{\partial}{\partial x^{B b}}\right\}$ will be denoted by $\nabla_{C c} q^{A a}$ and $\tilde{\nabla}_{C c} q^{A a}$. For each of the generators $(35)$ we define one of the tensors:

$$
\begin{aligned}
& \chi_{A a B b}^{(\mu)}=\sum_{E, F=1}^{N} P_{A a}^{E e} P_{B b}^{F f} \tilde{\nabla}_{E e} u_{F f}^{(\mu)} \\
& \omega_{A a B b}^{(\nu)}=\sum_{E, F=1}^{N} P_{A a}^{E e} P_{B b}^{F f} \tilde{\nabla}_{E e} v_{F f}^{(\nu)} .
\end{aligned}
$$

Pairs of indices $A a$ of these tensors are raised and lowered with the flat metric (33).

$\dagger$ For a generic discussion of a rigged $(n-m)$ dimensional manifold in an $n$-dimensional manifold endowed with a linear connection see Schouten 28 
From straightforward algebra on Eq. (21) written in the principal axis frame the following useful expression emerges:

$$
\sum_{A=1}^{N} m_{A}{\underset{\circ}{x^{A \mu}}}_{x_{\circ}^{A \nu}}^{A \nu} \frac{\delta^{\mu \nu}}{2} \sum_{\rho}\left(1-2 \delta_{\mu \rho}\right) I_{\rho} .
$$

By use of the above relation, together with Eqs. (35), (36) and (19), we compute the expressions for the $\chi^{(\mu)}$ and $\omega^{(\nu)}$ tensors in the principal axis frame in terms of the principal moments of inertia:

$$
\begin{aligned}
& \chi_{A \alpha B \beta}^{(\mu)}=0 \\
& \omega_{A \alpha B \beta}^{(\nu)}=-\frac{m_{B}}{\sqrt{I_{\nu}}} \epsilon_{\alpha \beta}^{\nu}\left(\delta_{A B}-\frac{m_{A}}{M}\right)
\end{aligned}
$$

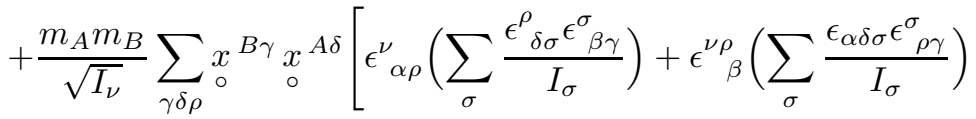

$$
\begin{aligned}
& \left.-\sum_{\lambda \mu \chi} \epsilon_{\lambda}^{\nu \rho}\left(\sum_{\sigma} \frac{\epsilon_{\alpha \delta \sigma} \epsilon_{\rho \mu}^{\sigma}}{I_{\sigma}}\right)\left(\sum_{\tau} \frac{\epsilon_{\chi \tau}^{\lambda} \epsilon_{\beta \gamma}^{\tau}}{I_{\tau}}\right)\left(\sum_{G=1}^{N} m_{G} \underset{\circ}{x}{ }_{\circ}^{G \mu} \underset{\circ}{G \chi}\right)\right] .
\end{aligned}
$$

The tensor transformation law gives the expression of $\omega^{(\nu)}$ in a generic frame:

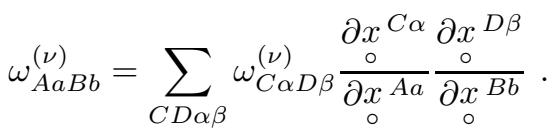

Similarly, $\chi_{A a B b}^{(\mu)}=0$ holds in any frame.

The tensor $\chi_{A a B b}^{(\mu)}$ being symmetric (it vanishes) is the extrinsic curvature of the hypersurfaces with normal vectors $u_{\mu}^{A a}$. By contrast, the tensor $\omega_{A a B b}^{(\nu)}$ is not hypersurface orthogonal, being antisymmetric. It is the vorticity tensor for the vectors $w_{\nu}^{A a}$. This antisymmetry originates in the transformation properties of $w_{(\nu)}^{B b}$ under reflections: it behaves as an axial (pseudo) vector rather then a polar (true) vector. The antisymmetry of the tensor $\omega_{A a B b}^{(\nu)}$ complicates the relation between the Riemann curvature tensors $\tilde{R}_{B b C c D d}^{A a}$ (here vanishing) and $R_{B b C c D d}^{A a}$, associated to the metrics $g_{A a B b}$ and $G_{A a B b}$, respectively.

We derive the required generalized Gauss equation as follows. Let $q^{B b}, r^{C c}$ and $s^{D d}$ be three arbitrary vectors and $p_{A a}$ an arbitrary one-form in the tangent and cotangent spaces of the space of orbits, respectively. The two Riemann tensors are defined as:

$$
\begin{aligned}
& \sum_{B=1}^{N} \tilde{R}_{B b C c D d}^{A a} q^{B b}=2 \tilde{\nabla}_{[C c} \tilde{\nabla}_{D d]} q^{A a} \\
& \sum_{B=1}^{N} R_{B b C c D d}^{A a} q^{B b}=2 \nabla_{[C c} \nabla_{D d]} q^{A a} .
\end{aligned}
$$

Evaluating the expression $\tilde{R}_{B b C c D d}^{A a} p_{A a} q^{B b} r^{C c} s^{D d}$, we get the desired relation between the Riemann curvature tensors of the metric $g$ on the $3 N$ dimensional space and of the metric $G$ on the rigged $(3 N-6)$ dimensional space:

$$
\sum_{B, E, F, G, H=1}^{N} \tilde{R}_{F f G g H h}^{E e} P_{E e}^{A a} P_{B b}^{F f} P_{C c}^{G g} P_{D d}^{H h} q^{B b}=2 \sum_{\nu} \omega_{[C c D d]}^{(\nu)}\left(\ell_{w_{(\nu)}} q^{A a}\right)
$$




$$
+\sum_{B=1}^{N}\left[R_{B b C c D d}^{A a}-2 \sum_{\nu}\left(\omega_{[C c}^{(\nu) A a} \omega_{D d] B b}^{(\nu)}-\omega_{[C c D d]}^{(\nu)} \omega_{B b}^{(\nu) A a}\right)\right] q^{B b},
$$

where $\ell_{w_{(\nu)}} q^{A a}$ denotes the Lie-derivative $\mathcal{L}_{w_{(\nu)}}$ of the vector $q^{A a}$, projected to the reduced space:

$$
\ell_{w_{(\nu)}} q^{A a}=P_{B b}^{A a} \mathcal{L}_{w_{(\nu)}} q^{B b} .
$$

The major inconvenience induced by the antisymmetric $\omega_{A a B b}^{(\nu)}$ tensors in (47) is the presence of the derivative term of $q^{B b}$. For generic riggings such a term should be absorbed in the definition of the Riemann tensor of the reduced space as described by Schouten [28]. However in our case the situation is simpler. As translations and rotations are symmetries of the system, the quantities defined on the space of orbits (in particular the components of the tangent vector $q^{A a}$ ) should not depend on the parameters of translations and rotations. In an adapted coordinate system the Lie-derivatives are partial derivatives with respect to these parameters, thus the Lie-derivative terms in (47) vanish. Because the vanishing of a tensorial quantity is a invariant statement, this result holds in arbitrary coordinate system as well. Therefore and by employing the flatness of the metric $g$, the expression of the Riemannian curvature tensor of the rigged space emerges purely in terms of the vorticity tensors $\omega^{(\nu)}$ :

$$
R_{B b C c D d}^{A a}=2 \sum_{\nu}\left(\omega_{[C c}^{(\nu) A a} \omega_{D d] B b}^{(\nu)}-\omega_{C c D d}^{(\nu)} \omega_{B b}^{(\nu) A a}\right)
$$

This is the desired generalized Gauss equation.

The curvature scalar is then readily found:

$$
R=\sum_{A, B, D=1}^{N} G^{B b D d} R_{B b A a D d}^{A a}=-3 \sum_{\nu} \omega_{B b}^{(\nu) A a} \omega_{A a}^{(\nu) B b} .
$$

Here $G^{B b D d}$ denotes the inverse metric of the rigged space. A lengthy, but straightforward computation, performed in the system of principal axes of inertia, employing the explicit expressions of the vorticity tensors (44) and the auxiliary relation (43) has given the scalar curvature in terms of the principal moments of inertia and the number of particles:

$$
R=6(N-2) \sum_{\nu} \frac{1}{I_{\nu}}-\frac{3}{2 I_{1} I_{2} I_{3}}\left(\sum_{\nu} I_{\nu}\right)^{2}
$$

$R$ being scalar, the above expression is independent of the particular frame employed for its evaluation.

The concise form (51) allows one to find out whether the manifold of orbits can be extended by the inclusion of the alligned configurations. The metric $G_{A a B b}$ extended there by a limiting process, is singular. The question arises, whether these line configurations represent coordinate or real singularities. Suppose that the system is passing through a sequence of configurations $\mathcal{C}_{n}$ toward the line configuration $\mathcal{C}_{\text {line }}$ with all particles aligned on the $x$-axis. There $I_{1}=0$ while $I_{2}=I_{3}=I$ are finite. The curvature scalar (51) behaves as

$$
\lim _{\mathcal{C}_{n} \rightarrow \mathcal{C}_{\text {line }}} R=\frac{6(2 N-5)}{I}+\frac{6(N-3)}{I_{1}} .
$$

Excepting the case $N=3$ the curvature scalar diverges together with the vanishing of $I_{1}$. Thus the line configurations are curvature singularities for $N \neq 3$. 


\section{Connections with molecular dynamics}

It is instructive to compare our description with a geometric treatment of the $N$-body problem in the language of fiber bundles, employed in both classical and quantum molecular dynamics. A recent review of this topic was given by Littlejohn and Reinsch [25]. In that approach the translational degrees of freedom are subtracted from the very beginning by the introduction of the $3 N-3$ Jacobi coordinates. When the configurations are not collinear, the remaining structure is identified with a principal fiber bundle with the structure group $S O(3)$. The orbits of this group are the fibers and the base space is what we have called the reduced configuration space (shape space in the language of (25)). Two kind of frames play an essential role in this approach: the space frame, which is an externally prescribed inertial frame, and the body frame, which can be attached to a flexible body in many ways. This arbitrariness in the choice of the body frame is a gauge freedom, any choice of gauge corresponding to a section of the fiber bundle. A gauge potential $A_{a}^{i}$ is introduced. The decomposition of the motion in rotational and vibrational part is gauge dependent, however the curvature form,

$$
B_{a b}^{i}=\frac{\partial A_{b}^{i}}{\partial q^{a}}-\frac{\partial A_{a}^{i}}{\partial q^{b}}-\epsilon_{j k}^{i} A_{a}^{j} A_{b}^{k},
$$

called also Coriolis tensor is gauge covariant. Here $\left\{q^{a}\right\}$ are generic coordinates in shape space and the indices $a, b$ symbolize the tensorial character of the Coriolis tensor with respect to changes in the shape space coordinates, while indices $i, j, k$ denote the components in some body frame. Thus the Coriolis tensor is a geometric object with $3 \times(3 N-6) \times(3 N-6)$ components.

A metric $G_{a b}$ in shape space is also introduced by means of the horizontal contribution to the kinetic energy. By a Kaluza-Klein type decomposition the Riemann tensor and curvature scalar associated to this metric are found, respectively:

$$
\begin{aligned}
& R_{a b c d}=\frac{1}{2}\left(B_{a b}^{i} I_{\circ} i j B_{c d}^{j}-B_{a[c}^{i} I_{\circ}|i j| B_{d] b}^{j}\right) \\
& R=\frac{3}{4} B^{i a b} I_{0}{ }_{i j} B_{a b}^{j} .
\end{aligned}
$$

In the second formula the indices $a, b$ were raised by the inverse of the (non-flat) metric $G_{a b}$.

Specializing to vanishing angular momentum leads to horizontal motions in the principal fiber bundle. It is thus not surprising, that we have exploited a somewhat simpler geometric structure in our analysis.

Our approach differs in many aspects, as we were discussing a purely relational theory. We cannot start from the concept of space frame. It would imply to monitor the evolution of the system with respect to some preexisting inertial system, which is not the case. By contrast, we have chosen a definite body frame for our computations in Sec. 4. This is the principal axis frame.

We have imposed constraints on the dynamics by means of Lagrange-multipliers, at the end of the reduction process arriving to a Jacobi-type action (29). The Lagrangian in [25, without the kinetic energy of the center of mass and specialized to vanishing angular momentum (when the vertical contribution to the kinetic energy about the center of mass vanishes) is

$$
L(q, \dot{q})=\frac{1}{2} G_{a b} \dot{q}^{a} \dot{q}^{b}-V(q) .
$$


By writing the Jacobi principle version of this action with the energy set to zero we can identify the metric $G_{a b}$ with our metric $G_{A a B b}$, expressed in a coordinate system adapted to the six generators.

By comparing our formulae (49) and (50) for the Riemann tensor and curvature scalar with Eqs. (54) and (55) respectively it is tempting to identify our vorticity tensor $\omega_{A a B b}^{(\nu)}$ with the Coriolis tensor. For this purpose first we write Eq. (55) in the principal axis frame:

$$
R=3 \sum_{\nu} \frac{\sqrt{I_{\nu}} B^{\nu a b}}{2} \frac{\sqrt{I_{\nu}} B_{a b}^{\nu}}{2}
$$

Second, we remark that unlike the Coriolis tensor, the vorticity tensors are defined as three geometric object with $3 N \times 3 N$ components. However by their definition (42) as projections we see that the vorticity tensors live in the $(3 N-6)$-dimensional reduced space (shape space). When coordinates adapted to this space are chosen, they will have equally $(3 N-6) \times(3 N-6)$ nonvanishing components. Third, indices $A a, B b$ are raised with the (inverse) flat metric $g^{A a B b}$, in contrast with the contravariant form of the Coriolis tensor in (57), where indices are raised with the nonflat metric $G^{a b}$. But there is no difference in raising the indices of $\omega_{A a B b}^{(\nu)}$ with the nonflat metric $G^{A a B b}$ either. This is because the two metrics differ only in terms homogeneous in the rigging vectors. When contracted with the vorticity tensor (a projected object) these difference terms vanish by virtue of Eqs. (41).

Thus we identify the set of the three vorticity tensors $\omega^{(\nu)}$ with $\sqrt{I_{\nu}} B^{\nu} / 2$ (no summation). The advantage in employing the vorticity tensors, which are defined on the (unreduced) configuration space is that their indices can be raised and lowered with the flat metric $g$.

\section{Concluding Remarks}

The study of the Barbour-Bertotti theory describing the Newtonian $N$ - particle system constrained by the vanishing of all constants of the motion revealed that a complete reduction at the Lagrangian level is possible whenever the particles are not along a line.

The reduced space emerged as the space of orbits of the Leibniz-group. Free motions are geodesics with respect to the Riemannian metric $G_{A a B b}$ while motions characterized by $V \neq$ const are geodesics of the conformally scaled metric $-2 V(x) G_{A a B b}$. The vorticity tensors of the generators of rotations were introduced and their expression in terms of the pricipal moments of inertia was derived.

The Riemann tensor and the curvature scalar associated with the metric $G_{A a B b}$ were given in terms of the vorticity tensors. The additional expression of the scalar curvature in terms of the principal moments of inertia allowed for a study of the collinear configurations. Curvature singularities arise in these configurations, unless the number of particles is three.

The Riemannian curvature tensor and the curvature scalar associated to the conformally scaled metric $\hat{G}_{A a B b}=-2 V(x) G_{A a B b}$, which applies to the case $V \neq$ const can be found by applying the relations among these objects for conformally related metrics 30. For illustration we give here the curvature scalar $\hat{R}$ associated to 
the metric $\hat{G}$ :

$$
\begin{aligned}
\hat{R}=\frac{1}{-2 V}\{R & -(3 N-7) G^{A a B b}\left[\nabla_{A a} \nabla_{B b} \log (-V)\right. \\
& \left.\left.+\left(\frac{3 N}{4}-2\right) \nabla_{A a} \log (-V) \nabla_{B b} \log (-V)\right]\right\} .
\end{aligned}
$$

For any definite choice of the potential $V$, such relations can be further exploited.

\section{Acknowledgments}

The description of the Barbour-Bertotti model in Sec. 2 is based on unpublished notes by Karel Kuchař. It is a pleasure to thank him for suggesting the problem and for useful references. The author is grateful for the warm hospitality of the relativity group at the Physics Department of University of Utah and for clarifying discussions with László Szabados and Mitchell McKain in the early stages of this work. The comments of the referees led to improvements in the presentation and they are acknowledged. This work was realized with the continued support of the NSF grant PHY-9734871, OTKA grants W015087 and D23744, the Eötvös Fellowship and the Soros Foundation.

\section{References}

[1] Newton I 1934 Principia ed F Cajori (UCLA Press, Los Angeles)

[2] Mach I 1957 The Science of Mechanics (Nelson, London)

[3] 1965 The Leibniz-Clark Correspondence ed H G Alexander (Manchester)

[4] Lynden-Bell D and Katz J 1995 Phys. Rev. D 527322

[5] Lynden-Bell D, Katz J and Bičak J 1995 Mon. Not. Roy. Astron. Soc. 272150

[6] Barbour J B 1994 Class. Quantum Grav. 112853

[7] Reißner H 1914 "Über die Relativität der Beschleunigung in der Mechanik" Physikalische Zeitschrift XV 371

[8] Reißner H 1915 "Über die Möglichkeit die Gravitation als unmittelbare Folge der Relativität der Trägheit abzuleiten" Physikalische Zeitschrift XVI 179

[9] Lynden-Bell D 1992 in Variable Stars and Galaxies, ed B Warner ASP Conf. Ser. Vol. 30 (Astronomical Society of the Pacific, San Francisco) p 1

[10] Barrow J D, Juskiewicz B R and Sonada D H 1985 Mon. Not. R. Astron. Soc. 213917

[11] Hawking S W 1969 Mon. Not. R. Astron. Soc. 142129

[12] Collins C B and Hawking S W 1973 Mon. Not. R. Astron. Soc. 162307

[13] Barbour J B and Bertotti B 1977 Nuovo Cimento B38 1

[14] Barbour J B and Bertotti B 1982 Proc. Roy. Soc. Lond. A382 295

[15] Lanczos C 1970 The Variational Principles of Mechanics (Toronto Univ. Press)

[16] Barbour J B and Smolin L 1988 Can quantum mechanics be sensibly applied to the universe as a whole? (Yale University preprint)

[17] Barbour J $\mathrm{B}$ and Smolin 1992 Extremal variety as the foundation of a cosmological quantum theory (hep-th/9203041)

[18] Rovelli C 1991 in Conceptual Problems in Quantum Gravity ed A Ashtekar et al (Birkhäuser, Boston)

[19] Gambini R and Mora P 1994 Intrinsic time and evolving Hilbert spaces in relational dynamical systems and quantum gravity (hep-th/9404169) (1994)

[20] Barbour J B 1994 Class. Quantum Grav. 112875

[21] Gergely L Á and McKain M The geometry of the Barbour-Bertotti theories II. The three body problem subsequent paper

[22] Baierlein R F, Sharp D H and Wheeler J A 1962 Phys. Rev. 1261864

[23] Bartnik R and Fodor G 1993 Phys. Rev. D 483596

[24] Giulini D 1999 J. Math. Phys. 402470

[25] Littlejohn R G and Reinsch M 1997 Rev. Mod. Phys. 69213 The metric G was denoted there by $g$. 
[26] Synge J L 1927 Philos. Trans. of the Royal Soc. of London 22631

[27] Sydłowski M 1999 J. Math. Phys. 403519

[28] Schouten J A 1954 Ricci-Calculus (Springer-Verlag)

[29] Littlejohn R G and Reinsch M 1995 Phys. Rev. A 522035

[30] Wald R M 1984 General Relativity Appendix D (The Univ. of Chicago Press) 